\title{
PROCESO DE CONSTRUCCIÓN DE LA AGENDA NACIONAL DE INVESTIGACIÓN SOBRE RECURSOS HUMANOS EN SALUD (RHUS) EN EL PERÚ, 2011 - 2014
}

\author{
Maricela Curisinche ${ }^{1, a}$, Martín Yagui ${ }^{1, b}$, Teresa Castilla ${ }^{1, c}$, \\ César Cabezas ${ }^{1, d}$, Giovanni Escalante ${ }^{2, e, f}$, María Casas ${ }^{1, g}$, Jorge Lucero $^{1, h, i}$
}

\begin{abstract}
RESUMEN
La Autoridad Sanitaria Nacional del Perú, como parte del proceso de implementación de las prioridades nacionales de investigación en salud en el 2010, desarrolló el proceso de construcción de la agenda nacional de investigación sobre recursos humanos en salud (RHUS). En un escenario de desafíos técnico-políticos nacionales e internacionales y bajo un enfoque participativo de alcance nacional, con actores clave del sistema de salud, formación de RHUS y cooperantes vinculados con el tema, se establece una agenda socialmente consensuada. El proceso consta de tres fases: 1. Revisión nacional de evidencias e información relevante sobre RHUS; 2 . Consulta a líderes de opinión y expertos temáticos y 3. Un espacio participativo (taller nacional) de deliberación, consenso y legitimación de la agenda. Finalmente se presenta la agenda compuesta por 30 temas de investigación sobre RHUS para ser desarrolladas en el período 2011-2014 y se plantean los retos y perspectivas para su implementación.
\end{abstract}

Palabras clave: agenda de investigación en salud, recursos humanos en salud, participación ciudadana, consenso: Perú (fuente: DeCS BIREME).

\section{PROCESS OF CONSTRUCTION OF THE NATIONAL RESEARCH AGENDA ON HUMAN RESOURCES IN HEALTH IN PERU 2011 - 2014}

\begin{abstract}
The National Health Authority of Peru, as part of the implementation of national priorities for health research in 2010 developed the process of building the national research agenda on health manpower (HM). In a scenario of technical challenges, national and international policy and under a nation-wide participatory approach with key stakeholders in the health system, training and aid HM linked to the subject, establishing a socially agreed agenda. Process consists of 3 phases: 1. National review of evidence and relevant information on RHUS, 2. Consultation with opinion leaders and subject experts, and 3. A collaborative space (national workshop) of deliberation, consensus and legitimacy of the agenda. Finally, we present the agenda consists of 30 research topics on health manpower, to be developed in the period 20112014, and raises the challenges and prospects for implementation.
\end{abstract}

Keys words: Health Research Agenda, Health Manpower, Citizen Participation, Consensus: Perú (source: MeSH NLM).

\section{INTRODUCCIÓN}

Los Recursos Humanos en Salud (RHUS) constituyen una preocupación mundial pues se ubican, en los últimos años, en el centro del debate global en salud, lo cual ha generado una agenda internacional de desarrollo de los RHUS ${ }^{(1-3)}$, con importantes retos y compromisos para los países, incluido el Perú (1), tales como los desafíos del Llamado a la Acción de Toronto para una Década de RHUS 2006-2015 (2), el Plan Regional de Acción de RHUS 2007-2015 (3), el Plan Andino de RHUS 2008-2015 ${ }^{(4)}$, la medición de las Metas Regionales de
RHUS 2007-2015 - OPS/OMS y en los países de la Región Andina ${ }^{(5)}$, y el Código de prácticas mundial sobre contratación internacional de personal de salud $2010{ }^{(6)}$, entre los principales.

En el Perú, se ha logrado establecer políticas, normas y planes ${ }^{(7)}$ de salud vinculados con las políticas de Estado. La Ley General de Salud (Ley 26842), la Política Nacional de Salud, la Política Nacional para el Desarrollo de los RHUS-2005 (8), el Plan Nacional Concertado y Descentralizado de Desarrollo de Capacidades en Salud 2010-2015 (9), la Línea Basal de

\footnotetext{
Instituto Nacional de Salud. Lima, Perú.

2 Organización Panamericana de la Salud. Lima, Perú.

a Enfermera Salubrista; ${ }^{\mathrm{b}}$ Médico Epidemiólogo; ${ }^{\mathrm{C}}$ Enfermera epidemióloga; ${ }^{\mathrm{d}}$ Médico Infectólogo; ${ }^{\mathrm{a}}$ Médico; ${ }^{\mathrm{f}}$ Magister de Ciencias de Salud Pública para países en vías de Desarrollo; ${ }^{g}$ Licenciada en Enfermería; ${ }^{\mathrm{h}}$ Biólogo; ' Doctor en Salud Pública.
} 
las Metas Regionales de RHUS en el Perú 2009 (10), así como la implementación del aseguramiento universal en salud ${ }^{(8,11-13)}$, la descentralización de la función salud a las regiones ${ }^{(14,15)}$ y la renovación de la atención primaria de la salud (14,16-18), constituyen desafíos relevantes que convergen como elementos trazadores de análisis en el proceso de identificación de la agenda.

La Autoridad Sanitaria Nacional (ASN) de investigación en salud, ha desarrollado un progresivo proceso de fortalecimiento de la gobernanza y rectoría de la investigación en salud en el último quinquenio, como una función esencial de la salud pública ${ }^{(19)}$. Las capacidades de rectoría se expresan en las mejoras en el marco legal, financiamiento, creación y sostenibilidad de recursos, definición de prioridades de investigación, producción y utilización de la investigación (11). De este modo, se contribuye a reducir las brechas entre la producción de investigaciones y la solución de problemas sanitarios prioritarios de la población y del sistema de salud, así como acrecentar el uso de la investigación en la formulación y evaluación de políticas, programas e intervenciones en salud ${ }^{(19-21)}$. Con la conducción del Instituto Nacional de Salud (INS) y la participación social de los actores vinculados con el sistema de salud, se logró identificar siete prioridades nacionales de investigación para el período 2010-2014 ${ }^{(22)}$. Entre ellas destacan las investigaciones para conocer los problemas de los recursos humanos en salud (RHUS) como primera prioridad nacional ${ }^{(22)}$.
En el 2010, se impulsa la implementación de las prioridades nacionales de investigación en salud, dirigidos a establecer las agendas de investigación por cada prioridad, en procesos participativos similares. Se concluye el 2010 con agendas consensuadas de tres prioridades nacionales de investigación: RHUS, salud mental y VIH; el 2011 se continuó con las demás (Figura 1).

Como se observa, este abordaje utiliza los fundamentos para la generación de políticas públicas como cursos de acción intencionados, democráticamente establecidos, con la finalidad de generar un valor público en salud, lo que constituye otro aporte al fortalecimiento del buen gobierno en salud ${ }^{(23)}$.

En tal sentido, la conducción de estos procesos se asumen en alianza estratégica con las ASN responsables de los problemas sanitarios que componen la prioridad nacional de investigación, puesto que ellos serán los principales usuarios de los resultados de las investigaciones en la generación/diseño e implementación de políticas, normas e intervenciones de alcance nacional (19,21). En RHUS, la ASN forma la Dirección General de Gestión del Desarrollo de los Recursos Humanos (DGGDRH) del Ministerio de Salud.

El presente artículo describe el contexto, la metodología y los resultados del proceso de construcción de la agenda nacional de investigación sobre RHUS en el

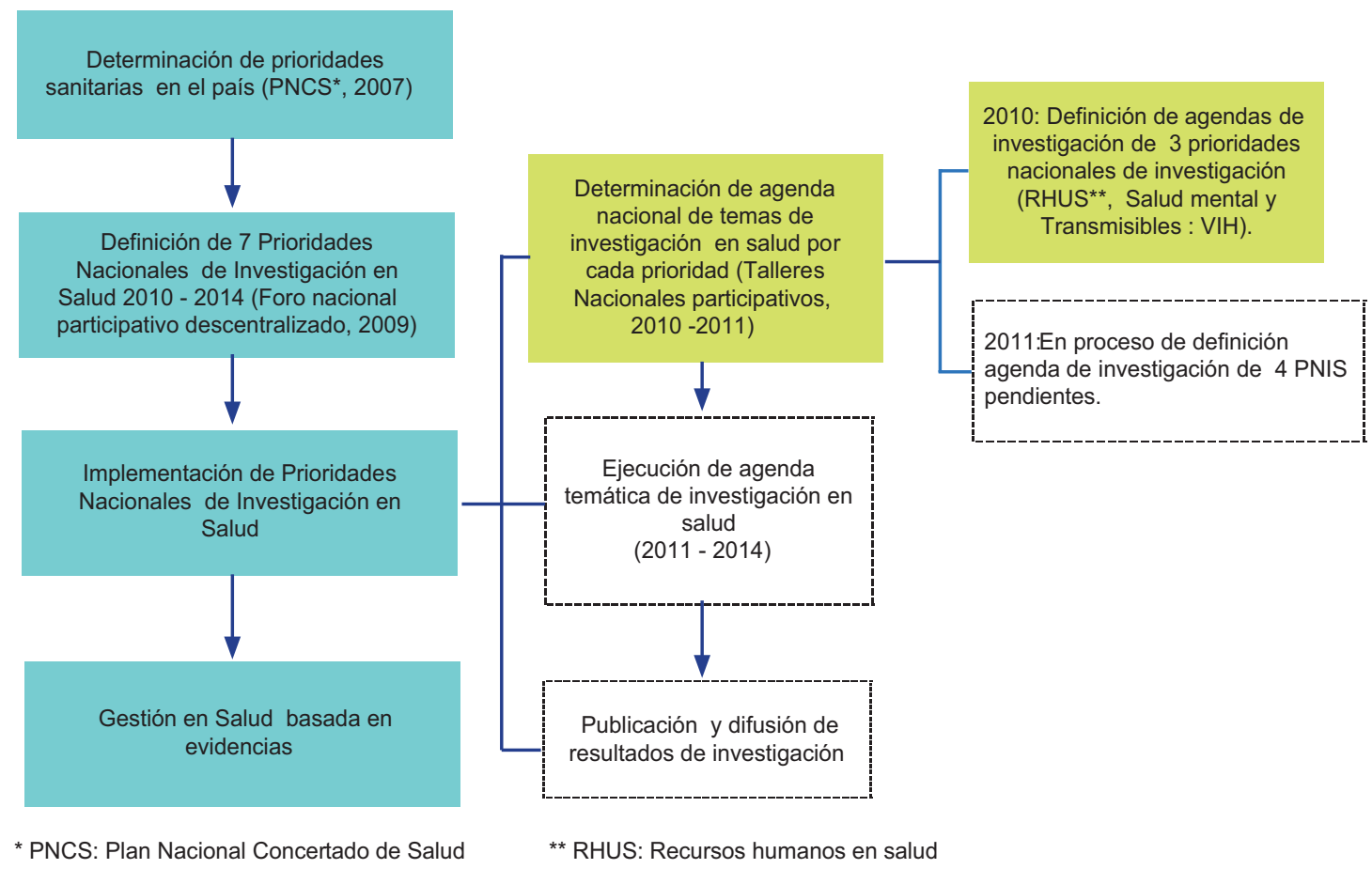

Figura 1. Proceso de implementación de prioridades nacionales de investigación en salud (PNIS) en el Perú, 2009 - 2014 
Perú, período 2011-2014. Señala el enfoque participativo, de amplia convocatoria nacional de actores sociales, y la metodología utilizada para su implementación en el corto y mediano plazo. Luego, presenta la agenda resultante, como tópicos de investigación específicos y trazadores a escala nacional, que generarán evidencias y respuestas a importantes vacíos e interrogantes sobre el problema de RHUS en el país ${ }^{(14,24-26)}$, todo ello será parte del insumo, para la ASN y para los gestores de la formación de RHUS, en la construcción de políticas e instrumentos de regulación (19-21) que mejoren la capacidad de respuesta del sistema de salud ${ }^{(21)}$.

\section{METODOLOGÍA DEL PROCESO}

Considerando que no existe un modo para establecer agendas de investigación sobre RHUS, se diseñó una metodología ad-hoc para el Perú, donde el enfoque de participación ciudadana ${ }^{(27,28,29)}$, la deliberación y el consenso social (30), sean el punto principal de dicha metodología, para así afirmar su legitimidad y viabilidad en el tiempo. El proceso constituye un encuentro constructivo de actores vinculados con la investigación, el desarrollo de RHUS, la provisión de servicios de salud, la formación de RHUS, los cooperantes, entre otros, en torno a la agenda de investigación, desde una perspectiva de inclusión y descentralización, con amplia convocatoria al sector público, privado y las regiones del país.

\section{FASES DEL PROCESO}

\section{FASE I: \\ REVISIÓN DE EVIDENCIAS \\ E INFORMACIÓN RELEVANTE}

Con el fin de contar con un panorama nacional sobre disponibilidad y vacíos en investigación sobre RHUS, para así contextualizar el problema y las necesidades de investigación en el país, se exploró diversas fuentes de información, recopilándose estudios de investigación y otras evidencias sobre RHUS en el Perú generados en los últimos diez años (2000 - 2010). Se examinó once fuentes de obtención de datos, donde Google Académi$\mathrm{co}$, la Biblioteca Virtual OPS-PERU y la Biblioteca Virtual de Salud BVS/BIBLIOMINSA, fueron las fuentes de referencias bibliográficas sobre investigaciones en RHUS realizados en el Perú.

\section{FASE II: \\ CONSULTA A LÍDERES DE OPINIÓN \\ Y EXPERTOS TEMÁTICOS}

Se realizó una fase de consulta técnica a líderes de opinión y expertos en RHUS, a fin de involucrarlos en el diseño y organización en el tema. Se aplicó un cuestionario estructurado con preguntas abiertas y cerradas, autoadministrado o por medio de entrevistas, para obtener información relevante sobre percepción, opinión y aportes respecto al tema y orientación en el proceso de identificación de la agenda de temas de investigación.

Para la consulta, se elaboró un directorio de líderes temáticos nacionales representantes de diversas instituciones de salud, de formación de RHUS, proyectos de cooperación, entre otros (EsSalud, Ministerio de Salud, directivos y docentes de pre y postgrado de universidades, directores regionales de salud, responsables de RHUS de proyectos de cooperación, investigadores de RHUS y expertos nacionales en el tema), lográndose consultar a 19 líderes.

\section{FASE III: PROCESO DE DELIBERACIÓN, CONSENSO Y LEGITIMACIÓN}

Generamos un espacio participativo de deliberación, consenso y legitimación de la agenda, a través del "Taller de Definición de la Agenda Nacional de Investigación sobre RHUS 2011-2014" conducido por el Instituto Nacional de Salud conjuntamente con la Dirección General de Gestión del Desarrollo de Recursos Humanos (DGGDRH).

\section{El taller se desarrolló en tres momentos:}

\section{Primer momento:}

Centrada en la contextualización, sensibilización y abogacía sobre los problemas de los RHUS en el país, así como la relevancia e implicaciones de la investigación en salud y en los RHUS, a nivel nacional.

\section{Segundo momento:}

Núcleo del Taller, centrado en la construcción y priorización de la agenda nacional de temas de investigación para resolver los problemas de RHUS. Los participantes agrupados en mesas temáticas, en dos rondas de deliberación y consenso alrededor del tema clave asignado, obtienen como productos, una propuesta de cinco temas relevantes de investigación sobre RHUS (primera ronda) y tres temas de investigación priorizados según criterios establecidos (segunda ronda). Considerando que no existe una metodología definida para establecer prioridades en investigación, el equipo técnico conductor decidió diseñar una, ad-hoc para el Perú, que a su vez constituya una contribución para los países de las Américas. La metodología adoptada se centró en la combinación de enfoques que fueron ajustados y adecuados a los objetivos del proceso, y que facilitaron de manera lógica, ágil y participativa, en espacios de deliberación y consenso, plantear, priorizar, determinar y sustentar 


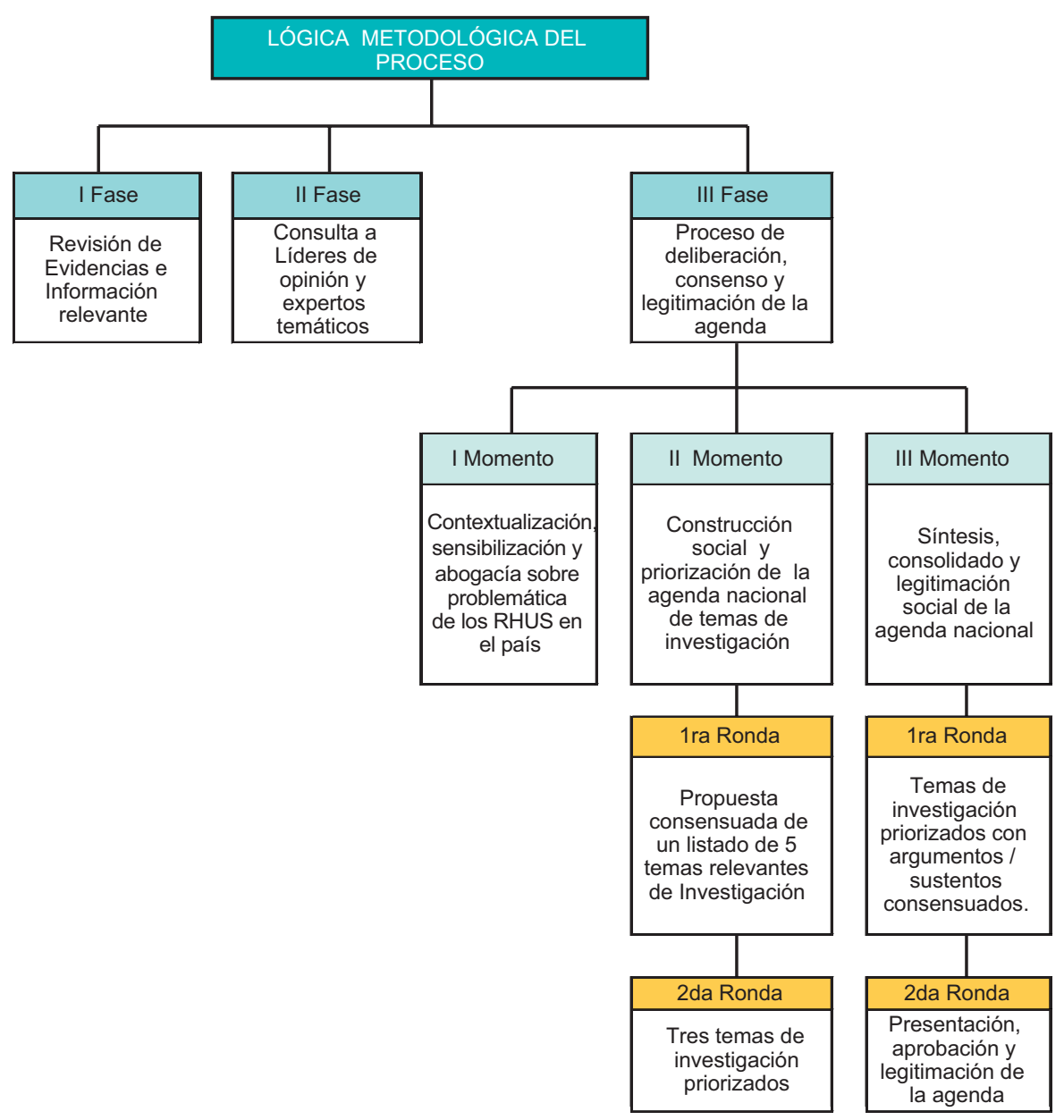

Figura 2. Construcción de la agenda nacional de investigación sobre RHUS 2011 - 2014.

la agenda de temas específicos de investigación sobre RHUS.

\section{Criterios de Priorización}

Para establecer los criterios de priorización, se consideró el enfoque basado en criterios políticos y técnicos (14) y los criterios definidos en el foro nacional de prioridades nacionales de investigación en salud (4), seleccionándose de este último los cuatro más relevantes y pertinentes, los cuales fueron adaptados al tema de RHUS:

- Magnitud del problema: qué tan grande y grave es el problema con relación a los otros.

- Importancia: efectos o consecuencias en el desempeño de los RHUS y del sistema de salud.

- Viabilidad: posibilidad de investigar desde el punto de vista político, técnico, financiero y operativo.

- Efecto en equidad: si se investiga el problema, probabilidad de impacto para atención de los segmentos más pobres de la población.

\section{Tercer momento:}

Comprende la síntesis, consolidado y legitimación de la agenda. Incluye dos rondas de deliberación y consenso, cuyos productos constituyen, los argumentos y sustentos de cada tema de investigación priorizado (primera ronda) y la aprobación del pleno de deliberantes de la agenda socializada y sustentada en plenaria por cada grupo (segunda ronda). En la plenaria general, los representantes de cada mesa temática socializan y sustentan los temas de investigación priorizados, los que con la aprobación del pleno pasan a formar parte de la agenda nacional de investigación para conocer los problemas de los recursos humanos en salud para el período 2011 - 2014 (Figura 2).

\section{ANÁLISIS DE DATOS}

Se desarrolló un aplicativo electrónico en formato Excel para el procesamiento del trabajo deliberativo y los productos finales de las nueve mesas. Este instrumento tuvo 
Tabla 1. Investigaciones sobre recursos humanos en salud en el Perú, según Contenido Temático. 2000 - 2010.

\begin{tabular}{lccc}
\hline \multicolumn{1}{c}{ Temas } & n & \% \\
\hline Desempeño laboral y desarrollo de capacidades & 27 & 36 \\
\hline Formación de pregrado de RHUS & 19 & 25 \\
\hline Calidad de la formación de RHUS & 11 & 15 \\
Formación de postgrado y segunda & 6 & 8 \\
especialización & 5 & 6 \\
\hline Aseguramiento universal y RHUS & 3 & 4 \\
Descentralización y RHUS & 2 & 3 \\
Atención primaria y RHUS & 2 & 3 \\
Migración de personal de salud & 0 & 0 \\
Salud y seguridad de los trabajadores de salud & 0 & 0 \\
Carrera sanitaria & 75 & 100 \\
\hline Total & & \\
\hline
\end{tabular}

Fuente: Lucero T. Jorge: Informe "Investigaciones sobre RHUS en Perú 2000-2010". Octubre $2010^{(31)}$

un papel relevante por su utilidad, facilitó el procesamiento de la información, así como el cumplimiento del trabajo en los tiempos establecidos. Este aplicativo fue instalado en los equipos asignados a cada una de las mesas temáticas. Se realizó un análisis descriptivo (frecuencias y porcentajes) de los hallazgos por fases del proceso.

\section{RESULTADOS}

\section{REVISIÓN DE EVIDENCIAS}

En la revisión nacional de evidencias sobre los RHUS, se encontró 75 investigaciones publicadas, la mayoría de estudios y publicaciones se concentran en desempeño laboral y desarrollo de capacidades, formación de pregrado y calidad de la formación de los RHUS (Tabla 1) ${ }^{(31)}$, la mayoría de estudios fueron descriptivas cuantitativas (60\%), luego los descriptivos cualitativos (19\%) y revisiones bibliográficas (19\%). Estos resultados evidencian insuficiente investigación e información respecto a temas trazadores sobre RHUS en el país.

\section{CONSULTA A LÍDERES DE OPINIÓN Y EXPERTOS TEMÁTICOS}

En la consulta técnica a expertos temáticos en RHUS, se obtuvo respuesta de 19 autoridades y profesionales clave, cuya información se organizó según los procesos de educación y trabajo, componentes del marco conceptual del "Campo de los RHUS". Se determinó nueve temas trazadores sobre RHUS en el país, que condujeron el proceso de identificación y priorización de la agenda de investigación.

En Educación en Salud los temas trazadores son: 1. Formación de pregrado de RHUS y 2. Formación de
Tabla 2. Percepción de líderes y expertos temáticos sobre problemas más importantes en el área de educación y trabajo en salud, Perú 2010.

\begin{tabular}{|c|c|c|}
\hline $\begin{array}{l}\text { Problemas más importantes en el área } \\
\text { de la educación en salud }\left({ }^{*}\right)\end{array}$ & $\begin{array}{l}\text { Frecuen- } \\
\text { cia }\end{array}$ & $\%$ \\
\hline Formación por competencias & 23 & 34 \\
\hline Gestión educativa & 11 & 16 \\
\hline Calidad educativa & 10 & 15 \\
\hline Formación con enfoque biomédico & 8 & 12 \\
\hline Regulación de las Instancias formadoras & 7 & 10 \\
\hline Campos clínicos sobrepoblados. & 3 & 4 \\
\hline Currículos de formación débiles en APS $\left({ }^{* *}\right)$ & 2 & 3 \\
\hline Tendencia a súper especialización médica & 2 & 3 \\
\hline $\begin{array}{l}\text { Ausencia de estudios de oferta y demanda } \\
\text { educativa }\end{array}$ & 1 & 1 \\
\hline Total & 67 & 100 \\
\hline $\begin{array}{c}\text { Problemas más importantes en el área } \\
\text { del trabajo en salud }\left(^{*}\right)\end{array}$ & Frecuencia & $\%$ \\
\hline Precarización del empleo & 10 & 20 \\
\hline Ausencia de carrera sanitaria & 8 & 16 \\
\hline $\begin{array}{l}\text { Condiciones de salud y seguridad en el } \\
\text { trabajo }\end{array}$ & 6 & 12 \\
\hline No existe gestión del desempeño & 6 & 12 \\
\hline No existe planificación de RHUS & 5 & 10 \\
\hline $\begin{array}{l}\text { Falta de perfiles de competencias labo- } \\
\text { rales }\end{array}$ & 4 & 8 \\
\hline $\begin{array}{l}\text { Bajas remuneraciones del personal de } \\
\text { salud. }\end{array}$ & 3 & 6 \\
\hline $\begin{array}{l}\text { No existe un sistema de motivación e } \\
\text { incentivos }\end{array}$ & 2 & 4 \\
\hline $\begin{array}{l}\text { Retención de profesionales en servicios } \\
\text { APS y zonas alejadas }\end{array}$ & 2 & 4 \\
\hline Elevada conflictividad laboral & 2 & 4 \\
\hline Complejidad de gestión de RHUS & 1 & 2 \\
\hline $\begin{array}{l}\text { Insuficiente dotación de especialistas y } \\
\text { personal profesional }\end{array}$ & 1 & 2 \\
\hline $\begin{array}{l}\text { Migración de profesionales de salud por } \\
\text { escasas oportunidades de empleo digno. }\end{array}$ & 1 & 2 \\
\hline Total & 51 & 100 \\
\hline
\end{tabular}

(*) Cada líder tuvo la opción de mencionar hasta cinco problemas. Fuente: Consulta a líderes y expertos temáticos

postgrado y segunda especialización de RHUS y en Trabajo en Salud se encuentran: 1. Aseguramiento universal y RHUS, 2. Atención primaria de salud y RHUS, 3. Desempeño laboral y desarrollo de RHUS, 4. Migración del personal de salud, 5. Gestión de la salud y seguridad en el trabajo, 6. Carrera sanitaria y 7. Descentralización y RHUS.

Respecto a la percepción de los problemas más importantes, en Educación en salud, destacan los temas de formación por competencias y gestión y calidad educativa como los más frecuentes, mientras que precarización del empleo, ausencia de carrera sanitaria, condiciones de salud y seguridad en el trabajo, gestión del desempeño y falta de planificación de RHUS, constituyen los 
Tabla 3. Propuestas de temas de investigación en el área de educación y trabajo en salud Perú 2010.

\begin{tabular}{|c|c|c|}
\hline $\begin{array}{l}\text { Temas de investigación en el área } \\
\text { de la educación en salud }\left({ }^{*}\right)\end{array}$ & $\begin{array}{l}\text { Frecuen- } \\
\quad \text { cia }\end{array}$ & $\%$ \\
\hline Calidad de la formación de RHUS & 15 & 30 \\
\hline Formación por competencias & 9 & 18 \\
\hline Campos clínicos para la formación & 4 & 8 \\
\hline $\begin{array}{l}\text { Experiencias de formación con enfoque } \\
\text { integral }\end{array}$ & 4 & 8 \\
\hline Experiencias de currículo flexible & 3 & 6 \\
\hline Formación de postgrado & 3 & 6 \\
\hline Seguimiento de egresados & 3 & 6 \\
\hline Articulación docencia servicio & 3 & 6 \\
\hline $\begin{array}{l}\text { Empleo de TICS en la formación de } \\
\text { RHUS }\end{array}$ & 2 & 4 \\
\hline Interculturalidad en la formación & 2 & 4 \\
\hline Deserción de personal capacitado & 1 & 2 \\
\hline Sedes de formación & 1 & 2 \\
\hline Total & 50 & \\
\hline $\begin{array}{c}\text { Temas de investigación en el área } \\
\text { del trabajo en salud }\left({ }^{*}\right)\end{array}$ & $\begin{array}{l}\text { Frecuen- } \\
\quad \text { cia }\end{array}$ & $\%$ \\
\hline Evaluación por desempeño e incentivos & 12 & 22 \\
\hline Equidad de distribución de RHUS & 7 & 13 \\
\hline Gestión de RHUS & 5 & 9 \\
\hline Precarización del trabajo & 5 & 9 \\
\hline Competencias de los trabajadores & 5 & 9 \\
\hline Calidad de atención & 4 & 7 \\
\hline $\begin{array}{l}\text { Generación de políticas desde servicios } \\
\text { de salud }\end{array}$ & 3 & 5 \\
\hline Salud y riesgos ocupacionales & 3 & 5 \\
\hline Migración de los profesionales de salud & 2 & 4 \\
\hline Oferta educativa y demanda laboral & 2 & 4 \\
\hline Satisfacción de usuarios & 2 & 4 \\
\hline Desarrollo de carrera sanitaria & 2 & 4 \\
\hline Conflictividad laboral & 1 & 2 \\
\hline Segunda especialización en salud & 1 & 2 \\
\hline Total & 55 & \\
\hline
\end{tabular}

(*) Cada líder tuvo la opción de precisar cuatro o más temas de investigación.

Fuente: Consulta a líderes y expertos temáticos

problemas más sentidos en Trabajo en salud. (Tabla 2). En la exploración de propuestas temáticas de investigación, en Educación en salud, se identifican la calidad de la formación de los RHUS y la formación por competencias, como más relevantes, siendo evaluación por desempeño e incentivos y equidad en la distribución de RHUS, los más propuestos en el área de Trabajo en salud (Tabla 3).

\section{PROCESO DE DELIBERACIÓN, CONSENSO YLEGITIMACIÓN}

\section{Respuesta de organizaciones invitadas}

Se logró una importante convocatoria al taller nacional, (118 participantes superior a lo previsto), entre representantes de la ASN; investigadores, gestores de RHUS y profesionales de los servicios de salud, de instituciones públicas como: EsSalud, Sanidad de las Fuerzas Armadas y Policía Nacional, hospitales e institutos especializados, delegados de las direcciones de salud de las regiones del país y Lima, así como representantes de instituciones de salud privados.

Otros actores importantes fueron: la OPS, los proyectos de cooperación internacional, el Comité Nacional de Docencia, Atención de Salud e Investigación (Consejo Nacional de Salud), Colegios Profesionales, Consejo Nacional de Ciencia y Tecnología, entre otros. Destacó, a su vez, la participación de directivos y docentes de pre y postgrado de universidades y representantes de las asociaciones de facultades de salud.

Finalmente, con el apoyo de 20 facilitadores y la participación activa de los deliberantes, se obtuvo la agenda nacional consensuada. Posteriormente oficializada por la máxima Autoridad Sanitaria con la Resolución Ministerial correspondiente.

\section{Producto del taller}

La Tabla 4 presenta la agenda compuesta por 30 temas de investigación por desarrollar entre el 20112014. Los seis primeros, vinculados con educación/ formación en salud y el resto orientados a los ejes de políticas-planeamiento de la dotación, carrera sanitaria, gestión del trabajo, gestión de las migraciones entre otros. Estos resultados apuntan a profundizar la comprensión de la problemática de los RHUS, evidenciándose coincidencias con los resultados de la línea basal de las Metas Regionales de RHUS en el Perú-2009 (10). Esta vez, desde un amplio consenso y perspectivas de sinergias para el desarrollo de las investigaciones.

\section{DISCUSIÓN}

La priorización de investigación en salud es considerada como parte clave de los esfuerzos necesarios para fortalecer los sistemas nacionales de investigación en salud. Los mecanismos de priorización son necesarios para facilitar el incremento armonizado de investigación en salud, sobre todo por los aspectos financieros y el impacto de las enfermedades bajo estudio. Los procesos 
Tabla 4. Agenda Nacional de Investigación sobre Recursos Humanos en Salud Perú 2011 - 2014*

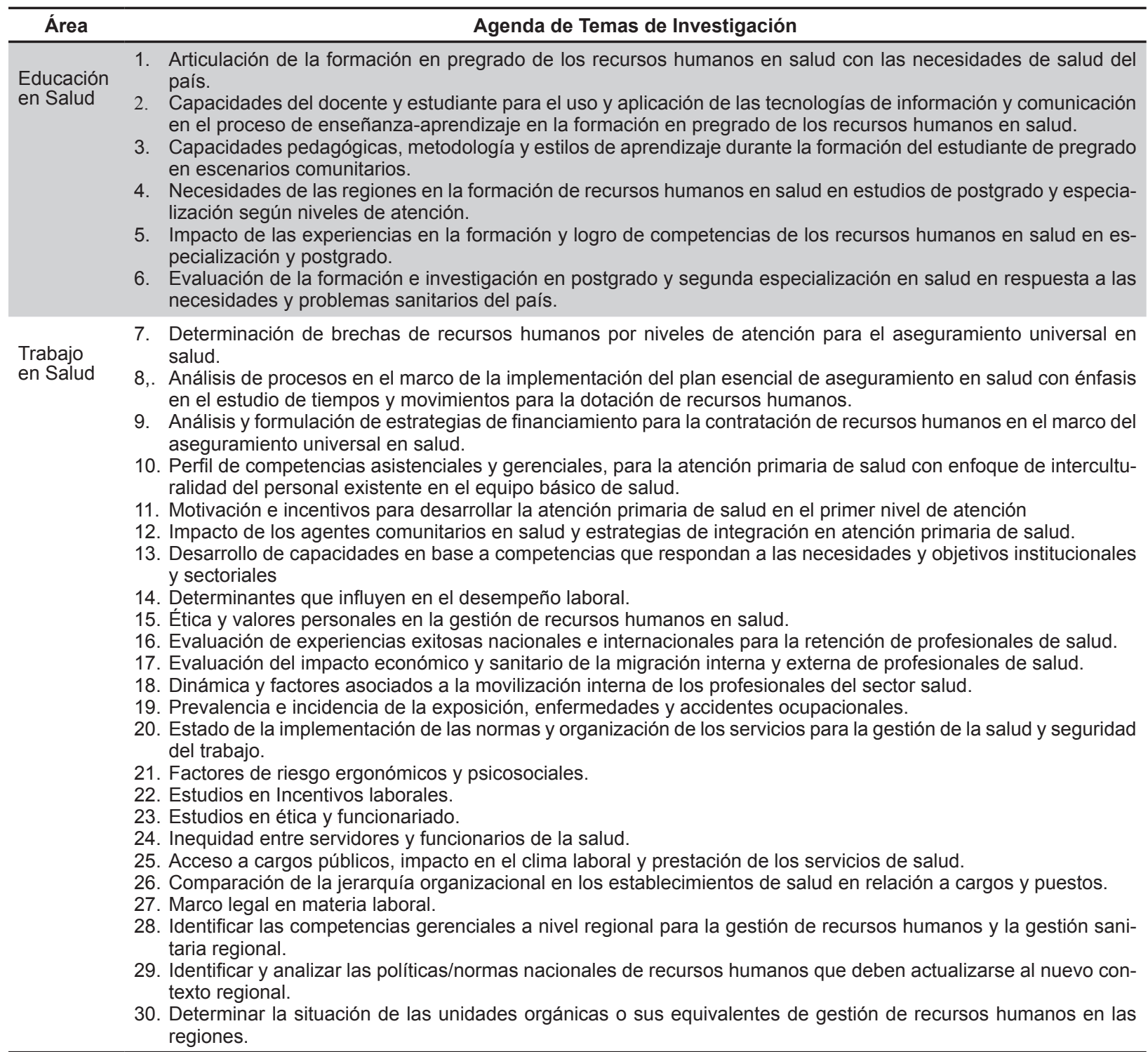

* Aprobada con Resolución Ministerial N. ${ }^{\circ}$ 212-2011/MINSA el 18 de marzo 2011 y Resolución Jefatural N. ${ }^{\circ}$ 269-2010-J-OPE/INS el 22 de octubre de 2010

de priorización de investigación en salud son asistidos por investigadores e implementadores de políticas para realizar una identificación efectiva de temas de investigación considerando su potencial beneficio en la salud pública ${ }^{(32)}$.

Los procesos participativos y representativos de la diversidad de sectores en varios niveles de organización cumplen una función en la mejora de los sistemas de salud. Entre los actores de los niveles organizacionales están: Salud - Educación - Gerencia de recursos humanos; dichos actores necesitan un horizonte común y aportan su experticia y metodología ${ }^{(33)}$.
Bajo dichos principios, se elaboró una agenda consensuada que constituye el cuerpo de potenciales investigaciones trazadoras a nivel país, con detalle temático y escenarios de aplicación precisos, que facilitarán su implementación, en un eje integrador desde el campo de la formación-capacitación, trabajo, gestión y regulación. La metodología fue participativa, con un buen nivel de convocatoria, con involucramiento activo y productivo de los actores, con convergencia de experticia y perspectivas de los actores, y con significativo consenso en torno a la agenda. El consenso logrado, legitima la agenda y compromete a los involucrados para futuros procesos sinérgicos de implementación de la agenda. Siendo clave, las 
alianzas estratégicas entre el sector salud, la formación/ capacitación de RHUS y los cooperantes.

El proceso fortalece el desempeño del sistema de salud y posiciona el liderazgo de la ASN en la gobernanza y rectoría de la investigación en salud; en un esfuerzo de armonización y alineamiento a favor de la construcción de políticas públicas, adopción de consensos y compromisos de los actores sociales. Dicho proceso participativo ha sido utilizado en el Instituto Nacional de Salud para establecer las prioridades nacionales y regionales de investigación en salud ${ }^{(6)}$. Dicho proceso de establecimiento de prioridades de investigación ha sido desarrollado en varios países, un estudio basado en la revisión de la literatura sobre procesos de establecimiento de prioridades, encontró una relativa falta de compromiso de los interesados, ninguna de las experiencias estudiadas tuvo un proceso sistemático u operativo para definir dichas prioridades y la mayoría de experiencias las prioridades se presentaron como categorías de enfermedad amplias y no se plantearon preguntas especificas de investigación ${ }^{(34)}$.

La necesidad de establecer una agenda de investigación para establecer preguntas específicas para los tomadores de políticas y los que ofrecen fondos para financiar investigación ha sido pobremente alineada con las prioridades nacionales, por lo que la lista de prioridades de investigación en salud debe ir acompañada con la elaboración de prioridades para la adjudicación de recursos en salud. Dicha elaboración de prioridades ha sido establecida mediante metodología participativa ${ }^{(35)}$. La metodología participativa ha sido utilizada también en elaboración de proyectos de salud específicos ${ }^{(36,37)}$. Sin embargo, no encontramos en la literatura la aplicación de una metodología participativa en la elaboración de una agenda de investigación en recursos humanos en salud.

En esta experiencia, además de la metodología planteada, se ha afianzado la articulación de dos instituciones de la ASN con funciones estratégicas en investigación en salud y RHUS (INS y DGGDRH). Articulación trascendente para acciones futuras en implementación de la agenda y utilización de la investigación en la propuesta de políticas sobre RHUS que optimicen la respuesta del sistema de salud.

La legitimación de la agenda por la máxima ASN, establece el aval y decisión política para su implementación e incorporación como política sanitaria de soporte a la gestión de RHUS en los diferentes niveles de gobierno sanitario.

Finalmente, se constituye un referente formal de acción común en investigación en RHUS, para los actores sociales y cooperantes involucrados en el tema a nivel nacional y regional.

\section{RETOS Y PERSPECTIVAS}

La construcción social de la agenda, le confiere una mirada optimista a futuro. Siendo su implementación la perspectiva inmediata de la ASN, constituye un reto substancial, sostener la convergencia, sinergia y participación activa de los actores sociales, en torno al desarrollo de mecanismos y estrategias que efectivicen la agenda en el corto y mediano plazo.

Por otro lado, la agenda plantea importantes perspectivas a la ASN en RHUS. Las evidencias por generar permitirán construir políticas e instrumentos de gestión, encaminados a transformar a los RHUS en elementos claves que mejoren la capacidad de respuesta del sistema de salud frente a importantes reformas sanitarias en el país, como descentralización en salud, aseguramiento universal, fortalecimiento de la atención primaria de la salud entre otros, y su vez como Estado, responder los retos internacionales sobre RHUS.

Para el Instituto Nacional de Salud, esto implica un mayor impulso de acciones estratégicas en proceso, dirigidos a consolidar el sistema nacional de investigación en salud (SNIS) y su gobernanza, fortalecer la gestión de la investigación en las regiones, generar competencias en investigación y gestión de la investigación en salud en los recursos humanos, establecer mecanismos para articular a los actores implicados en la investigación, generar alianzas estratégicas con el Consejo Nacional de Ciencia y Tecnología, el Observatorio Nacional de Recursos Humanos en Salud, entre otros.

Además, se requiere, la movilización de recursos del Estado en articulación con otras fuentes, establecer acuerdos gubernamentales en diferentes niveles del gobierno sanitario para asentar mecanismos de financiamiento (generación de fondos concursables, entre otras iniciativas), y a su vez, consolidar los esfuerzos sinérgicos del INS y la DGGDRH para atender la agenda.

La agenda dinamiza la perspectiva de trabajo conjunto entre actores sociales de instituciones formadoras, instituciones de salud, cooperantes entre otros; en torno a desarrollar una red de investigadores, establecer un registro especializado de investigaciones y promover la difusión sistemática de los resultados, que posibilite información oportuna de soporte a las decisiones.

Finalmente, la consolidación y posicionamiento de la ASN en investigación, como generadora de evidencias 
de políticas en salud, apertura de perspectivas de incorporar al Perú a la Red EVIPnet (Red mundial de evidencias de políticas de la OMS).

\section{AGRADECIMIENTOS}

A la Dirección General de Gestión del Desarrollo de los Recursos Humanos (DGGDRH) y su equipo técnico, por el compromiso asumido en el proceso conjuntamente con el INS. Un especial reconocimiento a los profesionales de la DGGDRH, el INS y otras instituciones que participaron como facilitadores del proceso, así como también, a los profesionales que participaron en condición de deliberantes.

\section{Contribuciones de Autoría}

Todos los autores participaron en la concepción del trabajo, revisión crítica y aprobación del documento final.

\section{Fuente de Financiamiento}

Instituto Nacional de Salud.

\section{Conflictos de Interés}

Los autores declaran no tener conflictos de interés.

\section{REFERENCIAS BIBLIOGRÁFICAS}

1. De los Ríos R. Gobernanza de la Investigación, función esencial de la salud pública. Elementos para la discusión. Washington, D.C.: OPS; 2005;

2. Yagui M, Espinoza M, Caballero P, Castilla T, Garro G, Yamaguchi LP et al. Avances y retos en la construcción del sistema nacional de investigación en salud en el Perú. Rev Peru Med Exp Salud Publica. 2010;27(3):367-72.

3. Organización Panamericana de la Salud. Política de investigación para la salud. Washington DC: OPS; 2009.

4. González D, Gómez-arias R, Vicente J, Malo M, Hernán$\operatorname{dez} \mathrm{T}$, Dávila F, et al. Retos de la investigación en salud pública hoy. Maracay: Instituto de Altos Estudios en Salud Pública; 2007.

5. Caballero P, Yagui M, Espinoza M, Castilla T, Granados A, Velásquez A et al. Prioridades Regionales y Nacionales de Investigación en Salud, Perú 2010 - 2014: Un proceso con enfoque participativo y descentralista. Rev Peru Med Exp Salud Publica. 2010;27(3):398-411.

6. Lahera E. Política y Políticas Públicas. Serie Políticas Sociales 95. Santiago de Chile: CEPAL; 2004.

7. GRADE. Estudio sobre los Recursos Humanos en Salud en Perú: Gestión, distribución, normatividad, regulación, condiciones laborales y salariales y Formación. Lima: GRADE; 2006.

8. Casas M. Proceso de Descentralización de la Gestión de los Recursos Humanos en Salud - Una Propuesta para su Operacionalización. Informe de Consultoría. Lima: OPS; 2005.
9. Proyecto AMARES. Estudio de disponibilidad y distribución de los recursos humanos en salud en las DIRESAS Ayacucho, Huancavelica y DISA Andahuaylas. Lima: AMARES; 2006.

10. Casas, M. Condiciones de trabajo y salud de los trabajadores de la salud en Argentina, Brasil, Costa Rica y Perú. Informe Perú. Lima: OPS; 2009.

11. Organización Panamericana de la Salud. Metas regionales en materia de recursos humanos para la salud 20072015. Washington, D.C.: OPS; 2007.

12. Organismo Andino de Salud, Organización Panamericana de la Salud. Plan Andino de Recursos Humanos 2008 - 2015. Quito: OPS; 2008.

13. Organización Panamericana de la Salud. Llamado a la acción de Toronto 2006-2015. Hacia una década de recursos humanos en salud para las Américas. Canadá: OPS; 2005.

14. Organización Panamericana de la Salud. Plan regional de acción de recursos humanos para la Salud, 2007-2015. Washington, D.C.: OPS; 2007.

15. Organización Panamericana de la Salud. Resultados de la primera medición de las metas regionales de RHUS 2007-2015 en la Región Andina, 2010. Lima: OPS; 2010.

16. Organización Mundial de la Salud. Código de prácticas mundial sobre contratación internacional de personal de salud 2010. 63 ${ }^{\text {a }}$ Asamblea Mundial de la Salud. Washington, D.C.: OPS; 2010.

17. Ministerio de Salud del Perú. Plan Nacional Concertado de Salud. Lima: MINSA; 2007.

18. Ministerio de Salud del Perú. Lineamientos de política nacional para el desarrollo de los recursos humanos en salud. Lima: MINSA; 2005.

19. Ministerio de Salud del Perú. Plan nacional concertado y descentralizado de desarrollo de capacidades en salud 2010 - 2015. Lima: MINSA; 2010.

20. Ministerio de Salud del Perú. Línea basal de las metas regionales de recursos humanos en salud en el Perú 2009. Lima: MINSA; 2010.

21. Ugarte $\mathbf{O}$. Aseguramiento universal en salud en el Perú. Rev Peru Med Exp Salu Publica. 2009;26(2):133.

22. Castro J. Hacia el aseguramiento universal en salud en el Perú. Rev Peru Med Exp Salud Publica. 2009;26(2):232-35.

23. Ministerio de Salud del Perú. Sistematización del proceso de implementación del monitoreo y evaluación de la descentralización en salud en el nivel regional orientado a mejorar el desempeño de la función Salud. Lima: MINSA; 2010.

24. Organización Panamericana de la Salud. Renovación de la Atención Primaria de Salud en las Américas. Washington, D.C.: OPS; 2005.

25. Suárez-Bustamante, M. La medicina familiar en el Perú: evolución y desafíos Rev Peru Med Exp Salu Publica. 2008;25(3):309-15

26. Zelada M, Mayta P. ¿Estamos preparados para la atención primaria del adulto mayor? Rev Peru Med Exp Salu Publica. 2006;23(1):67-9.

27. Martínez E. La gestión del conocimiento en políticas públicas en salud y participación social. Rev CES Med 2007;21(Supl 1):101-108. 
28. Van Hove EP. Participación ciudadana. Madrid: Green Cross España; 2010.

29. Sanhueza A. Participación ciudadana en la gestión pública. Santiago de Chile: Corporación Participa; 2004.

30. Valencia J. La gestión pública en el gobierno por deliberación. VII Congreso Internacional del CLAD sobre la Reforma del Estado y de la Administración Pública. Lisboa, Portugal, 8-11 Oct. 2002,

31. Lucero J. Investigaciones sobre RHUS en Perú 2000-2010 [Informe]. Lima: INS/OGITT; 2010.

32. Viergever R, Olifson S, Ghaffar A, Terry R. A checklist for health research priority setting: nine common themes of good practice. Health Res Policy Syst. 2010,8:36.

33. Parent F, Fromageot A, Coppieters Y, Lejeune C, Lemenu D, Garant M, et al. Analysis of adequacy levels for human resources improvement within primary health care framework in Africa. Health Res Policy Syst. 2005,3:8.

34. Tomlinson M, Chopra M, Hoosain N, Rudan I. A review of selected research priority setting processes at national level in low and middle income countries: towards fair and legitimate priority setting. Health Res Policy Syst. 2011,9:19.

35. Ranson K, Law T, Bennett S. Establishing health systems financing research priorities in developing countries using a participatory methodology. Soc Sci Med. 2010;70(12):193342.

36. Naylor P, Wharf J, Blair L, Green L, O Connor B. Evaluating the participatory process in a community-based heart health project. Soc Sci Med. 2002;55(7):1173-87.

37. Chung K, Lounsbury D. The role of power, process, and relationships in participatory research for statewide HIV/ AIDS programming. Soc Sci Med. 2006;63(8):2129-40.

Correspondencia: Maricela Curisinche Rojas Dirección: Cápac Yupanqui 1400, Lima11, Perú. Teléfono: (511) 617-6200 anexo 2179

Correo electrónico: mcurisinche@ins.gob.pe

\section{Consulte las ediciones anteriores de la Revista Peruana de Medicina Experimental y Salud Pública en www.scielo.org.pe Scitito Peri}

\section{FE DE ERRATAS}

Rev Peru Med Exp Salud Publica. 2010; 27(4): 557

Dice:

Claudia Zevallos-Palacios

Debe decir:

Claudia Zeballos-Palacios 